Instituto Internacional de Investigación y Desarrollo Tecnológico Educativo INDTEC, C.A.

DOI: https://doi.org/10.29394/Scientific.issn.2542-2987.2020.5.17.5.100-121

OAI-PMH: http://www.indteca.com/ojs/index.php/Revista Scientific/oai

Artículo Original / Original Article

\title{
SCImago: Un portal que deben conocer las universidades para posicionarse y alcanzar prestigio científico
}

\author{
Autores: Eddymar María Flores Nessi \\ Universidad Politécnica Territorial del Zulia, UPTZ \\ eddymarf.nessi@gmail.com \\ Zulia, Venezuela \\ https://orcid.org/0000-0003-1786-2283 \\ Derwis Antonio Sulbarán Sandrea \\ Universidad Politécnica Territorial del Zulia, UPTZ \\ dersulba@gmail.com \\ Zulia, Venezuela \\ https://orcid.org/0000-0001-6025-561X \\ Gisemar Ninoska Rojas de Ricardo \\ Universidad Nacional Experimental "Rafael María Baralt", UNERMB \\ gisemarninoska@gmail.com \\ Zulia, Venezuela \\ https://orcid.org/0000-0002-1723-3340
}

\section{Resumen}

Esta investigación constituye un análisis de los portales de internet de SCImago Journal \& Country Rank y SCImago Institutions Rankings, bases de datos en el que se incluyen indicadores de la actividad científica utilizados para evaluar la productividad de las universidades o instituciones dedicadas a la investigación, así como todas aquellas organizaciones encargadas de editar revistas científicas. Se fundamentó en los planteamientos de Rodríguez, Socorro y Espinoza (2019); Túñez-López, Valarezo-González y Marín-Gutiérrez (2014), entre otros. La metodología es de tipo documental-bibliográfica, se consultaron los portales de SCImago, así como artículos científicos e investigaciones referentes al tema de estudio. Estos portales ofrecen al usuario una serie de datos, herramientas y servicios de útil importancia para evaluar el desempeño de las instituciones gestoras de investigación, también, permiten medir el impacto de la actividad científica de los países a nivel mundial; se caracterizan por su fácil acceso y disponibilidad en la web, por lo que es importante que las universidades conozcan los indicadores científicos que se manejan en estos portales, con el fin de escalar posiciones dentro de los rankings investigativos, y les permita alcanzar prestigio científico ante comunidad nacional o internacional. indexación.

Palabras clave: base de datos; publicación científica; divulgación científica;

Cómo citar este artículo:

Flores, E., Sulbarán, D., \& Rojas, G. (2020). SClmago: Un portal que deben conocer las universidades para posicionarse y alcanzar prestigio científico. Revista Scientific, 5(17), 100121, e-ISSN: 2542-2987. Recuperado de: https://doi.org/10.29394/Scientific.issn.2542$\underline{2987.2020 .5 .17 .5 .100-121}$

Fecha de Recepción: 10-03-2020
Fecha de Aceptación: 11-06-2020
Fecha de Publicación: 05-08-2020 
OAI-PMH: http://www.indteca.com/ojs/index.php/Revista_Scientific/oai

Artículo Original / Original Article

\title{
SCImago: A portal that universities must know to position themselves and achieve scientific prestige
}

\begin{abstract}
This research constitutes an analysis of the Internet portals of SCImago Journal \& Country Rank and SCImago Institutions Rankings, databases that include indicators of scientific activity used to evaluate the productivity of universities or institutions dedicated to research, as well like all those organizations in charge of publishing scientific magazines. It was based on the statements of Rodríguez, Socorro and Espinoza (2019); Túñez-López, Valarezo-González and Marín-Gutiérrez (2014), among others. The methodology is of a documentary-bibliographic type, the SCImago portals were consulted, as well as scientific articles and research referring to the study topic. These portals offer the user a series of data, tools and services that are useful for evaluating the performance of research management institutions, and also allow measuring the impact of scientific activity in countries worldwide; They are characterized by their easy access and availability on the web, so it is important for universities to know the scientific indicators that are used in these portals, in order to climb positions within the research rankings, and allow them to achieve scientific prestige before national or international community.
\end{abstract} indexing.

Keywords: database; scientific publication; science popularization;

\footnotetext{
How to cite this article:

Flores, E., Sulbarán, D., \& Rojas, G. (2020). SCImago: A portal that universities must know to position themselves and achieve scientific prestige. Revista Scientific, 5(17), 100-121, e-ISSN: 2542-2987. Recovered from: https://doi.org/10.29394/Scientific.issn.2542-2987.2020.5.17.5.100-121
}

Date Received: 10-03-2020
Date Acceptance:

$11-06-2020$
Date Publication: 05-08-2020 


\section{Introducción}

Las universidades están llamadas a la construcción y producción de nuevos conocimientos, a través del trabajo inter y multidisciplinario, propiciando una cultura de investigación que le permita a estas instituciones alcanzar estándares científicos. Para esto es necesario que dentro de las universidades se apueste a la formación de investigadores (docentes o estudiantes de pre y postgrados), asimismo, se debe forjar el conocimiento en diversas áreas estratégicas o campos específicos de las ciencias; con el fin de solidificar alianzas, establecer vínculos con otras instituciones científicas, y mostrar el talento interesado por la investigación e innovación.

En este orden de ideas, las universidades se consideran los principales escenarios para llevar a cabo eventos de carácter científico, los cuales permiten difundir documentos como artículos, trabajos o proyectos de investigación, entre otros productos resultantes de las labores científicas y de innovación. En relación con esto, Martínez (2017), afirma que: "difundir el conocimiento científico y tecnológico, a través de los resultados originales, producto de investigaciones científicas, que representen una contribución para el desarrollo de la ciencia y la tecnología" (pág. 6).

Por su parte, Arechavala (2011): considera que la productividad depende no sólo de la formación y características personales de sus investigadores, sino del contexto organizacional e institucional en el que trabajan lo que determina fuertemente los patrones de productividad. Por lo que las universidades deben encaminarse a formar en la investigación y mediante la investigación, de manera que se alcance el desarrollo y la consolidación de la capacidad científica-tecnológica de las naciones.

En este sentido, es importante que las universidades y todas aquellas instituciones dedicadas a llevar a cabo labores de investigación conozcan y manejen algunos de los indicadores, herramientas y portales que evalúan la calidad de los productos de investigación. Destacando, que uno de los 
diferentes indicadores que permiten evaluar la productividad científica es el índice o factor de impacto proporcionado por SCImago.

Paralelamente, Rodríguez, Socorro y Espinoza (2019a): hacen referencia a que el uso del portal "SClmago Journal Rank (SJR) que influye en el desempeño de la universidad, su divulgación y reconocimiento por los docentes. Acercar el uso del SJR a la actividad académica constituye una alternativa para interpretar los avances científicos y tecnológicos [...]" (pág. $60)$.

En función a esto, este artículo constituye un análisis del sitio web de SCImago, conformado por SCImago Journal \& Country Rank (SJR) y SCImago Institutions Rankings (SIR). Los cuales forman parte de la plataforma desarrollada por el grupo SCImago, disponible públicamente en la web para la consulta de datos y herramientas relacionadas con una serie de indicadores cienciométricos que permiten analizar, comparar, y evaluar la productividad científica de instituciones encargadas de llevar a cabo investigación, desarrollo experimental e innovación.

Estos portales ofrecen al usuario de forma práctica e integrada, el acceso a diversos datos relacionados con dominios científicos, permitiendo realizar un análisis cuantitativo de la producción científica referente a la publicación de documentos o artículos como: memorias de eventos nacionales e internacionales; edición de libros o textos científicos publicados en revistas o medios de difusión, u otro órgano editorial bajo la modalidad escrita o electrónica.

El portal SCImago, permite visualizar la producción científica en las diferentes regiones del mundo, como África, Región Asiática, Europa Oriental, Latinoamérica, Medio Oriente, América del Norte, Región del Pacífico y Europa Occidental. Se tiene que, SCImago Journal \& Country Rank (SJR), publica anualmente el ranking de revistas y países, ordenando los mismos en base a la productividad científica reflejada en cantidad de artículos o documentos 
publicados. Por su parte, SCImago Institutions Rankings (SIR), permite clasificar las instituciones universitarias y organizaciones de investigación en relevancia con los altos índices científicos.

Es importante destacar, que este estudio busca describir el portal SCImago, herramienta que debe darse a conocer dentro de las universidades y todas aquellas instituciones editoras de revistas científicas, con el fin de que las mismas, alcancen credibilidad científica y logren posicionarse en los rankings SCImago. La divulgación de estos portales permitirá mejorar los artículos de los investigadores, sobre todo para aquellos que deseen que sus publicaciones alcancen visibilidad y prestigio científico.

\subsection{SCImago}

El portal SCImago, fue desarrollado por un grupo de investigación ubicado en España, conformado según SClmago Journal \& Country Rank (SJR, 2020a): por el "Consejo Superior de Investigaciones Científicas (CSIC), Universidad de Granada, Extremadura, Carlos III (Madrid) y Alcalá de Henares, dedicado al análisis, representación y recuperación de información mediante técnicas de visualización" (párr. 3).

El investigador principal, director y fundador del Grupo SClmago es Félix De Moya-Anegón, quien ha dirigido importantes proyectos contando con la colaboración de otros investigadores de la región iberoamericana. De MoyaAnegón, Bustos-González, Chinchilla-Rodríguez, Corera-Álvarez, LópezIllescas y Vargas-Quesada (2014), afirman que SCImago:

Ha desarrollado y adaptado metodologías tanto para el análisis de grandes dominios científicos, así como para estudiar sectores, regiones, instituciones e investigadores. Entre sus proyectos más destacados está SCImago Journal and Country Rank [...] SClmago Institutions Rankings [...] y Shape of Science [...] (pág. 7). 


\subsection{SCImago Journal \& Country Rank (SJR)}

El portal toma nombre del índice SJR desarrollado por el grupo SCImago. Para Rodríguez, Socorro y Espinoza (2019b), se destacan que: "el índice SJR se calcula a partir de las citas recibidas por las revistas objeto de análisis durante un periodo de 3 años, para ello se le otorga mayor importancia a las citas que proceden de artículos en revistas de alto prestigio [...]" (pág. 61). En esa misma línea, el sitio web de SClmago Journal \& Country Rank (SJR, 2020b), especifica lo siguiente:

Se incluyen las revistas y los indicadores científicos de países desarrollados, estos indicadores pueden usarse para evaluar y analizar dominios científicos. Las revistas se pueden agrupar por área temática (27 áreas temáticas principales), categoría temática (313 categorías temáticas específicas) o por país/revista y el año (desde 1999 hasta 2018). Los datos de citas provienen de más de 34.100 títulos de más de 5.000 editores internacionales y métricas de desempeño de 239 países en todo el mundo (párr. 1).

Para conocer la información manejada en el portal SCImago Journal \& Country Rank (SJR), se accede al sitio web de scimagojr.com. Una vez en la página de inicio se observan en la parte superior, el menú principal que incluye opciones para acceder a los datos como: Ranking de Revistas "Journal Rankings", Ranking por Países "Country Rankings", Herramientas "Viz Tools", Ayuda "Help" e Información sobre el portal "About Us". En referencia al tema, se incluye un buscador que permite ubicar una revista o el nombre de alguna publicación, a través título de la revista o el Número Internacional Normalizado de Publicaciones Seriadas (ISSN), dependiendo el análisis o los datos que requiera el usuario del portal se selecciona el icono de preferencia.

\section{a). Ranking de Revistas a través de SClmago}

Si el usuario requiere realizar la búsqueda de los rankings mundiales de revistas científicas en general, se selecciona Journal Rankings dentro del sitio 
web; seguidamente este nos lleva de forma rápida al ranking de revistas; luego el portal muestra un menú de navegación desplegable, como se visualiza en la figura 1 , en el que se pueden ingresar diversos parámetros de acuerdo a lo que requiera el usuario, filtrando la búsqueda por: área temática, categorías temáticas específicas, regiones o país de estudio, tipo de documento publicado (revistas, libros, memorias, entre otros) y año de consulta, con datos que van desde el año 1999 hasta el año 2018.

Figura 1. Menú de navegación desplegable para el ingreso de los datos de búsqueda dentro del Portal SCImago Journal \& Country Rank (SJR).

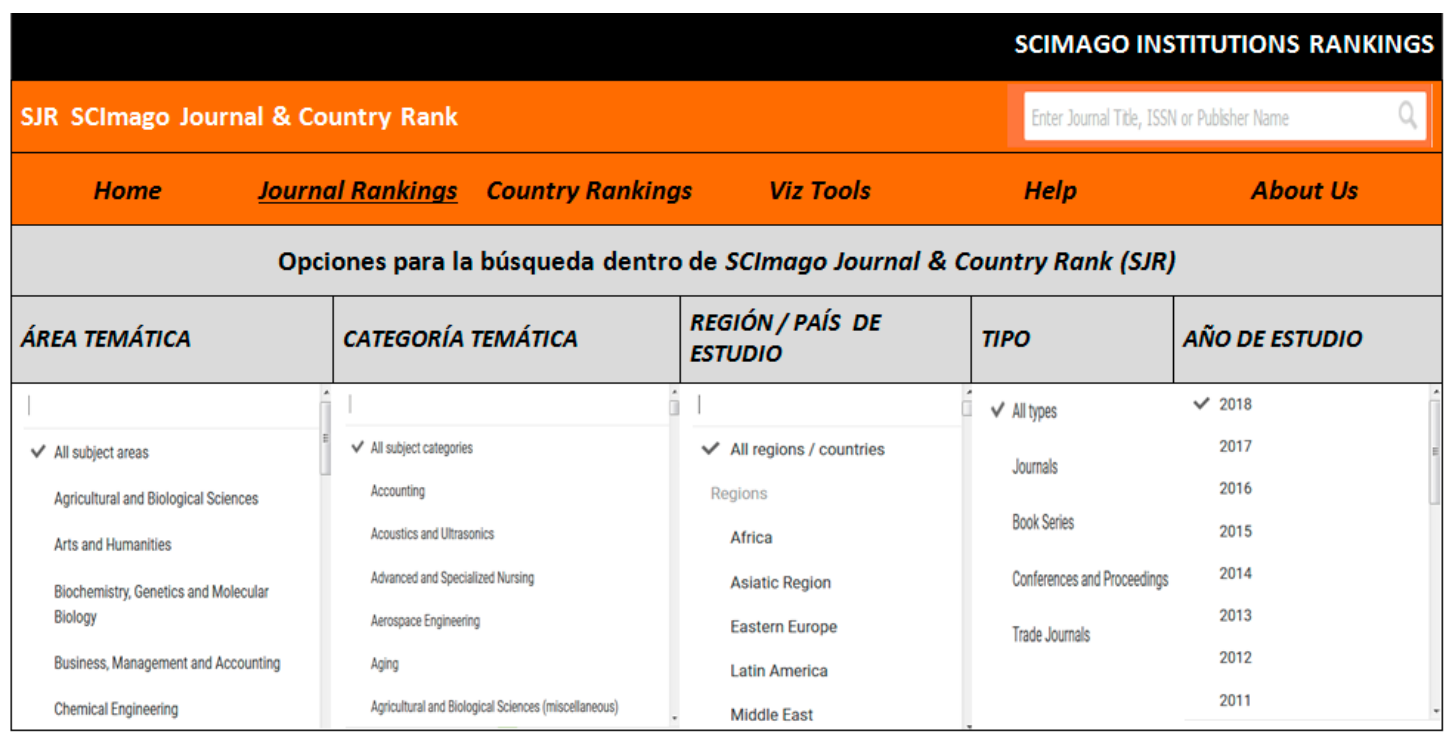

Fuente: SCImago Journal \& Country Rank (SJR, 2020).

De acuerdo a la búsqueda que requiera el usuario, los resultados son generados en forma de tabla en los que se organizan las revistas de acuerdo a un conjunto de indicadores: Índice SJR y cuartiles, Índice H, documentos totales publicados, documentos totales publicados en los últimos tres años, referencias totales incluidas en los artículos publicados, citas totales recibidas en los últimos tres años, documentos citables en los últimos 3 años, citas por documento en los últimos 2 años; también se muestra el país e institución que 
edita la revista y otros indicadores científicos que evalúan la calidad y el prestigio de la revista. Igualmente, el portal permite descargar estos datos a través de un fichero en formato de Microsoft Excel, para un posterior análisis del usuario.

Con relación a lo anterior, Rodríguez, Socorro y Espinoza (2019c), indican que: "en SCImago Journal \& Country Rank a partir del índice SJR se identifica cuál es el cuartil en que se ubica la revista y su factor de impacto en un área específica" (pág. 63). El cuartil es un indicador que permite evaluar una revista dentro del total de revistas en un área específica. Para esto se divide en cuatro cuartiles el listado de revistas en relación con todas las de su área. Señalando a Rodríguez, Socorro y Espinoza (2019d), el cuartil se identifica con la letra $Q$ y a través de colores, los cuales se precisan de la siguiente manera:

[...] Los colores van desde el verde para señalar el Q1, el amarillo para Q2, el naranja para Q3 y el rojo para Q4, con lo que se completa los cuatro cuartiles. Los cuartiles representan el $25 \%$ del total de revistas por lo que se hace corresponder con cada uno de ellos el Q1 es el de mayor SJR o mayor factor de impacto (pág. 63).

Además, Túñez-López, Valarezo-González y Marín-Gutiérrez (2014), plantean que el índice $\mathrm{H}$ : "es un indicador bibliométrico para medir la actividad investigadora y el impacto de su difusión, ya que equivale al dígito que equipara cuantitativamente las publicaciones de una revista o de un autor y las citas que éstas han obtenido" (pág. 897). Por lo que, un índice h=20 significa que hay un mínimo de 20 publicaciones que han recibido cada una de ellas al menos 20 citas.

Por ejemplo, si un usuario desea conocer el ranking de Revistas en el área Multidisciplinaria de la Región Latinoamericana, se ingresan estos datos en el menú desplegable, indicado en el portal y los resultados generados son enlistados de acuerdo los indicadores mencionados anteriormente, tal como 
se expresa en la tabla 1; y por último, el portal muestra las primeras revistas en el área Multidisciplinaria ubicadas en el SJR.

Tabla 1. Ranking de Revistas Multidisciplinarias en Latinoamérica (Elaborado a partir de datos obtenidos en SJR).

\begin{tabular}{|c|c|c|c|c|c|c|}
\hline Título & $\begin{array}{l}\text { SJR } \\
\text { Cuartil }\end{array}$ & Índice $\mathbf{H}$ & $\begin{array}{c}\text { Documentos } \\
\text { Publicados }\end{array}$ & $\begin{array}{c}\text { Documentos } \\
\text { Publicados } \\
\text { (Últimos } 3 \\
\text { años) }\end{array}$ & País & $\begin{array}{l}\text { Institución } \\
\text { editora de } \\
\text { la Revista }\end{array}$ \\
\hline $\begin{array}{c}\text { Anais da } \\
\text { Academia } \\
\text { Brasileira de } \\
\text { Ciencias } \\
\text { (ISSN: } \\
00013765)\end{array}$ & $\begin{array}{l}0,36 \\
\text { Q1 }\end{array}$ & 51 & 304 & 654 & Brasil & $\begin{array}{c}\text { Academia } \\
\text { Brasileira de } \\
\text { Ciencias }\end{array}$ \\
\hline $\begin{array}{c}\text { Brazilian } \\
\text { Archives of } \\
\text { Biology and } \\
\text { Technology } \\
\text { (ISSN: } \\
\text { 15168913) } \\
\end{array}$ & $\begin{array}{c}0,278 \\
\text { Q2 }\end{array}$ & 40 & 48 & 292 & Brasil & $\begin{array}{l}\text { Instituto de } \\
\text { Tecnología } \\
\text { do Parana }\end{array}$ \\
\hline $\begin{array}{l}\text { Interciencia } \\
\text { (ISSN: } \\
3781844 \text { ) }\end{array}$ & $\begin{array}{c}0,169 \\
\text { Q3 }\end{array}$ & 32 & 130 & 342 & Venezuela & $\begin{array}{l}\text { Asociación } \\
\text { Interciencia }\end{array}$ \\
\hline $\begin{array}{c}\text { Revista } \\
\text { Lasallista de } \\
\text { Investigación } \\
\text { (ISSN: } \\
\text { 17944449) }\end{array}$ & $\begin{array}{l}0,148 \\
\text { Q4 }\end{array}$ & 5 & 18 & 126 & Colombia & $\begin{array}{c}\text { Corporación } \\
\text { Universitaria } \\
\text { Lasallista }\end{array}$ \\
\hline
\end{tabular}

Fuente: SCImago Journal \& Country Rank (SJR, 2020).

Como se puede observar en la tabla 1, se tiene la Revista Anais da Academia Brasileira de Ciencias ubicada en el primer lugar de la región de Latinoamérica con mayores índices de productividad científica en el área Multidisciplinaria para el 2018 con un total de 304 documentos publicados; la misma se encuentra en el cuartil Q1 con un alto índice del impacto, posicionándose dentro de los mejores estándares del ranking SCImago.

Del mismo modo, el portal de SClmago Journal \& Country Rank (SJR, 2020c): permite analizar cada revista por separado, para la cual el usuario sólo debe hacer clic sobre el título de la revista y seguidamente se muestra un perfil individual en el que se incluyen una serie de gráficos que muestran la evolución de los indicadores científicos. Para el caso del ejemplo mencionado anteriormente, se analizará el perfil individual de la revista Anais da Academia 
Brasileira de Ciencias, explicado en la tabla 2, el cual muestra lo información relativa a los indicadores que miden la productividad de esta revista para el año 2018.

Tabla 2. Perfil individual de la revista Anais (Elaborado a partir de datos obtenidos en SJR).

\begin{tabular}{|l|l|}
\hline \multicolumn{2}{|c|}{ ANAIS DA ACADEMIA BRASILEIRA DE CIENCIAS } \\
\hline País de Procedencia & Brasil \\
\hline Área y categoría de análisis & Multidisciplinaria \\
\hline Institución Editora & Academia Brasileira de Ciencias \\
\hline ISSN & 00013765 \\
\hline $\begin{array}{l}\text { La Revista Anais da Academia Brasileira de ciencias es la revista oficial de la Academia } \\
\text { Brasileña de Ciencias y se ha publicado sin interrupciones desde 1929. Los volúmenes se } \\
\text { publican en marzo, junio, septiembre y diciembre. }\end{array}$ \\
\hline \multicolumn{2}{|c|}{ Índice H (año) } \\
SJR (año)
\end{tabular}

Fuente: SCImago Journal \& Country Rank (SJR, 2020).

\section{b). Ranking de países a través de SCImago}

Si el usuario quiere realizar un análisis de la producción científica mundial, tomando como referencia el número de documentos publicaciones en los diversos países o regiones del mundo, se selecciona dentro de la pantalla principal de inicio del portal SJR, en la categoría de Country Rankings, que al igual que el ranking de revistas, se muestra un menú de navegación desplegable para ingresar los parámetros y filtrar la búsqueda de acuerdo: área temática, categorías temáticas específicas, regiones o país de estudio, y año de consulta o periodo total para el análisis, con datos que van desde el año 1999 al año 2018. Una vez ingresados los datos preestablecidos por el usuario, los resultados son generados en forma de tabla, organizando los países de acuerdo con el número de documentos publicados, citas, autocitas, citas por documento e índice $\mathrm{H}$; por otra parte, todos estos datos pueden ser descargables a través de un fichero en formato en Microsoft Excel, para el posterior análisis del usuario. 
Tomando como ejemplo la Producción Científica por países en el área Multidisciplinaria de la Región Latinoamericana durante el periodo 1996- 2018, como se observa en tabla 3 , para los países de acuerdo al número total de documentos publicados, en el cual Brasil ocupa el primer lugar en la región con mayor producción científica para el área anteriormente especificada, detrás de México, Chile, Argentina, Colombia, Venezuela, Panamá, Puerto Rico, Perú y Ecuador, conjunto de países que ocupan los 10 primeros lugares del ranking de SCImago.

Tabla 3. Producción científica por países en el área Multidisciplinaria de la Región Latinoamericana 1996-2018 (Elaborado a partir de datos obtenidos en SJR).

\begin{tabular}{|c|c|c|c|c|c|c|c|}
\hline Ranking & País & $\begin{array}{c}\text { Documentos } \\
\text { Publicados }\end{array}$ & $\begin{array}{c}\text { Documentos } \\
\text { citados }\end{array}$ & $\begin{array}{c}\text { Citas } \\
\text { Recibidas }\end{array}$ & Autocitas & $\begin{array}{c}\text { Citas por } \\
\text { documento }\end{array}$ & $\begin{array}{c}\text { Índice } \\
\mathbf{H}\end{array}$ \\
\hline $\mathbf{1}$ & Brasil & 8923 & 8536 & 219767 & 46589 & 24,63 & 210 \\
\hline $\mathbf{2}$ & México & 2785 & 2660 & 123160 & 10094 & 44,22 & 160 \\
\hline $\mathbf{3}$ & Argentina & 1593 & 1501 & 91304 & 6770 & 57,32 & 139 \\
\hline $\mathbf{4}$ & Chile & 1405 & 1320 & 73541 & 6619 & 52,34 & 135 \\
\hline $\mathbf{5}$ & Colombia & 1055 & 1004 & 31473 & 1731 & 29,83 & 73 \\
\hline $\mathbf{6}$ & Venezuela & 1038 & 954 & 25410 & 1887 & 24,48 & 60 \\
\hline $\mathbf{7}$ & Panamá & 338 & 281 & 38492 & 2502 & 113,88 & 105 \\
\hline $\mathbf{8}$ & Puerto Rico & 300 & 279 & 25392 & 601 & 84,64 & 63 \\
\hline $\mathbf{9}$ & Perú & 244 & 226 & 19067 & 804 & 78,14 & 65 \\
\hline $\mathbf{1 0}$ & Ecuador & 193 & 163 & 9991 & 447 & 51,77 & 38 \\
\hline
\end{tabular}

Fuente: SCImago Journal \& Country Rank (SJR, 2020).

Al igual que la sección anterior de Journal Rankings, dentro del ranking por países el portal permite analizar cada país por separado, para lo cual el usuario sólo debe hacer clic sobre enlace del nombre del país para obtener datos del perfil individual, presentado en la tabla 4 , en el cual se incluyen una serie de datos sobre los indicadores científicos durante el periodo 1999-2018. 
Tabla 4. Perfil individual por país que ofrece el portal (Elaborado a partir de datos obtenidos en SJR).

\begin{tabular}{|c|c|c|c|}
\hline \multicolumn{4}{|c|}{ BRASIL } \\
\hline \multicolumn{4}{|c|}{$\begin{array}{c}\text { Enlace SIR Ranking que Ileva directamente a visitar el sitio web de SCImago } \\
\text { Institutions Rankings }\end{array}$} \\
\hline \multirow{2}{*}{\multicolumn{4}{|c|}{$\begin{array}{c}\text { Opciones de Búsqueda para analizar el país para todas las áreas temáticas } \\
\text { abordadas en el portal o por cada área temática específica. }\end{array}$}} \\
\hline & & & \\
\hline Índice $\mathrm{H}$ & $\begin{array}{l}\text { Documentos } \\
\text { Publicados }\end{array}$ & Citas Recibidas & $\begin{array}{l}\text { Citas por } \\
\text { Documento }\end{array}$ \\
\hline 530 & 938352 & 10225275 & 10.90 \\
\hline
\end{tabular}

Fuente: SCImago Journal \& Country Rank (2020).

Como se puede apreciar en la figura anterior, el perfil individual por revista en SCImago Journal \& Country Rank (SJR, 2020d): permite analizar el país para todas las áreas temáticas abordadas en el portal o por cada área temática específica; también se muestran otros indicadores como: Índice $H$, documentos, citas, citas por documento, entre otros indicadores. También, dentro del perfil individual el portal muestra debajo del nombre del país el enlace SIR Ranking, que lleva directamente a visitar el sitio web de SCImago Institutions Rankings, como medio para conocer y analizar todas aquellas instituciones ubicadas en ese país y que se dedican a llevar a cabo labores investigativas.

\subsection{SCImago Institutions Rankings (SIR)}

El portal SCImago Institutions Rankings (SIR, 2020): se encarga de clasificar todas las instituciones de educación universitaria e instituciones centradas en investigación, a partir de indicadores científicos (investigación, resultados de innovaciones, impacto social a través de su visibilidad en la web) que evalúan el nivel y la calidad investigativa.

Para conocer la información manejada de este portal, se accede al sitio web scimagoir.com. En la página de bienvenida del portal se ubica el menú 
principal que incluye Inicio "Home", "Rankings" y Metodología "Methodology"; también se incluye un buscador que permite colocar el nombre de la institución para un análisis individual, en el cual se muestra la evolución de los indicadores de productividad científica desde el año 2009 hasta el año 2020.

De igual forma, dentro de la página de bienvenida, se ofrece información general del portal, como se expone en la figura 2, así como la clasificación de las mejores instituciones y con más altos niveles de productividad científica para el período o año actual; también se incluye una Interfaz de Visualización que permite acceder a datos e información como: Mapa de País "Country Map", Mapa de Instituciones "Institutions Map", Distribución por países "Distribution By Country", Instituciones ubicadas en el SCImago a nivel global "SIR Globe" y evolución del ranking "Rank Evolution".

Figura 2. Información que contiene la Página de inicio de SCImago Institutions Rankings (SIR).

\begin{tabular}{|l|l|}
\hline SCIMAGO \\
INSTITUTIONS RANKINGS \\
RANKINGS
\end{tabular}

Fuente: SCImago Institutions Rankings (SIR, 2020).

La interfaz de visualización permite estudiar y ver con detalle a través de mapas y gráficos, todas aquellas instituciones con altos estándares 0 
excelentes desempeños en materia de investigación e innovación, su vinculación con el sector socioproductivo, y midiéndose a través de indicadores la producción y difusión científica de estas instituciones. Por otro lado, dentro de la página de inicio se incluye un enlace para visualizar y explorar el Informe Anual sobre la Clasificación de las instituciones universitarias iberoamericanas SIR Iber Reports, en función del número de documentos publicados y de acuerdo a los datos aportados por Scopus.

En conformidad con De Moya, Herrán-Páez, Bustos-González, CoreraÁlvarez, Tibaná-Herrera y Rivadeneyra (2019):

Desde el año 2009 SCImago Research Group publica anualmente dos informes: A) SIR World que muestra la actividad científica en el mundo [...] B) SIR Iber que muestra la actividad científica exclusivamente de Andorra, España, Portugal y los países de Latinoamérica [...]" (pág. 5).

Figura 3. Ingreso de los datos para la búsqueda dentro del Portal SCImago Institutions Rankings (SIR).

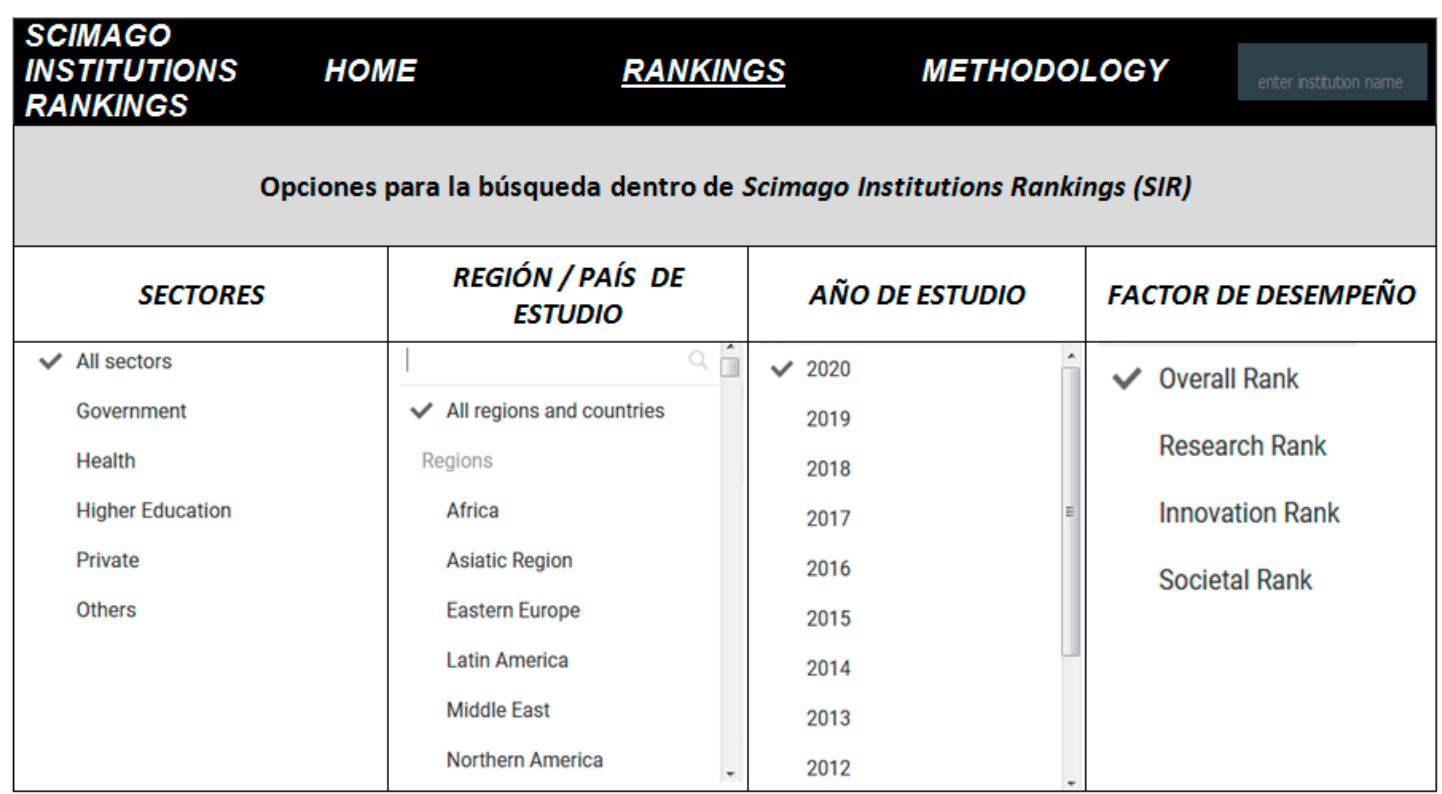

Fuente: SCImago Institutions Rankings (SIR, 2020). 
En otro orden de ideas, si el usuario quiere realizar un análisis de las universidades y todas aquellas instituciones gestoras de la investigación de acuerdo a la clasificación de SCImago, solo debe seleccionar en el menú principal del portal, la pestaña Rankings, inmediatamente la plataforma dirige de forma rápida al buscador al ranking de instituciones del mundo, como se presenta en la figura 3, luego se muestra un menú desplegable en el que se pueden ingresar diversos parámetros de acuerdo a lo que requiera el, filtrando la búsqueda por: sectores (Educación Superior o Universitaria, Gobierno, Salud, Sector privado y otros), regiones o país de estudio, año de consulta con datos que van desde 2009 hasta el 2020, y por último el rango o factor de desempeño (investigación, innovación o impacto social).

Luego de que se ingresen estos datos mencionados anteriormente, los resultados son generados a través de una tabla, que enlista las instituciones universitarias, institutos y/o centros de investigación de acuerdo a los resultados y características científicas que los hacen posicionarse dentro de altos niveles de investigación de acuerdo a su producción científica, en este sentido, el portal permite descargar todos estos datos a través de un fichero en formato Microsoft Excel para que usuario realice un análisis más profundo.

También, el portal permite realizar una comparación entre instituciones, para esto el usuario solo debe marcar en la casilla de selección que aparece al lado izquierdo del nombre de la institución que desea comparar; destacando que solo la plataforma permite seleccionar hasta seis instituciones para establecer dicha comparación. Esta comparación permite analizar el desempeño y evolución de estas instituciones en base a los factores de investigación, innovación e impacto social.

Por último, tomando como ejemplo el ranking de instituciones universitarias en la Región de Latinoamérica para el año actual 2020, expuesto en la tabla 5, una vez ingresados estos datos en el portal SIR, se muestran a través de una tabla todas aquellas instituciones universitarias ubicadas en la 
región antes mencionada, así como su posición con respecto al ranking mundial del portal SCImago.

Es importante destacar que el portal muestra un total de 160 posiciones para la región Latinoamericana, dentro de los cuales se ubican más de 100 universidades brasileiras con altos índices de productividad científica. Sin embargo, para efectos de análisis solo se muestran las 10 primeras instituciones ubicadas en este ranking, tomando en cuenta los tres factores de desempeño que abarca el portal (investigación, innovación o impacto social).

Tabla 5. Instituciones de Latinoamérica ubicadas en el Ranking SCImago (Elaborado a partir de datos obtenidos en SIR).

\begin{tabular}{|c|c|c|c|c|}
\hline $\begin{array}{l}\text { RANKING } \\
\text { LATAM }\end{array}$ & $\begin{array}{l}\text { RANKING } \\
\text { GLOBAL }\end{array}$ & INSTITUCIONES & \multicolumn{2}{|c|}{ PAÍS } \\
\hline 1 & 61 & Universidad de Sao Paulo & $\theta$ & Brasil \\
\hline 2 & 302 & Universidad Nacional Autónoma de México & - & México \\
\hline 3 & 336 & $\begin{array}{c}\text { Universidad Estadual Paulista Julio de } \\
\text { Mesquita Filho }\end{array}$ & $\theta$ & Brasil \\
\hline 4 & 379 & Universidad Estadal de Campinas & $\theta$ & Brasil \\
\hline 5 & 407 & Universidad Federal de Rio de Janeiro & $\theta$ & Brasil \\
\hline 6 & 445 & Universidad Federal de Minas Gerais & $\theta$ & Brasil \\
\hline 7 & 448 & Universidad Federal do Rio Grande do Sul & $\theta$ & Brasil \\
\hline 8 & 473 & Universidad de Chile & & Chile \\
\hline 9 & 489 & Pontificia Universidad Católica de Chile & & Chile \\
\hline 10 & 503 & Universidad de Buenos Aires & 0 & Argentina \\
\hline
\end{tabular}

Fuente: SCImago Institutions Rankings (SIR, 2020).

Se puede observar en la tabla anterior que entre estas instituciones existen seis universidades brasileiras, dos universidades chilenas, una universidad mexicana y una argentina, todas estas ubicadas dentro de las mejores instituciones con altos índices de producción científica. Es importante mencionar que la Universidad de Sao Paulo es la primera institución universitaria que se mantiene en este lugar desde el año 2009, de acuerdo con los datos reflejados en el portal de SCImago Institutions Rankings. 


\section{Metodología}

Este artículo sigue una metodología de tipo documental-bibliográfica, se recurrió al análisis de contenido definida por Hernández, Fernández y Baptista (2014): como "una técnica para estudiar cualquier tipo de comunicación de una manera "objetiva" y sistemática [...]" (pág. 251). Para efectos del análisis, se revisaron los sitios web de SCImago, conformados por SCImago Journal \& Country Rank (SJR) y SCImago Institutions Rankings (SIR), además de artículos publicados en revistas científicas. Los criterios de búsqueda se centraron en el tema de investigación, y los parámetros fueron previamente seleccionados, a través de las palabras claves como: SCImago, producción científica, indicadores científicos, entre otros.

\section{Reflexiones Finales}

El portal SCImago conformado SCImago Journal \& Country Rank y SCImago Institutions Rankings representan una herramienta muy importante para las universidades, es necesario que dentro de estas instituciones se propicie una cultura de investigación que involucre el estudio de indicadores cienciométricos que miden el impacto y la productividad científica, lo que permitiría a los investigadores e instituciones editoras de revistas científicas evaluar su aporte científico, económico y social, con el fin de que estas instituciones logren posicionarse dentro de los rankings SCImago, ya que el impacto de sus contribuciones les permitiría alcanzar prestigio científico a escala nacional e internacional.

El uso de estos portales permitirá a los investigadores dentro de las instituciones universitarias estudiar el desempeño de las revistas científicas en los cuales desean que sus publicaciones alcancen visibilidad e impacto científico. Se destaca que, el sitio web de SClmago pone a disposición una serie de indicadores que miden el impacto y la producción científica; lo que le permite al usuario estudiar, analizar, comparar diferentes espacios de la 
actividad científica. También, los interesados podrán analizar el rendimiento de las diferentes universidades e instituciones editoras de revistas indexadas, en cuanto al número de artículos publicados, referencias por artículos, citas recibidas, entre otros indicadores que les permitirán a las instituciones compararse con la competencia, así como estudiar las políticas que se tienen en cuanto a las actividades científicas, con el fin de que establezca una metodología para el reimpulso de capacidad científica-tecnológica y la mejora continua de estas instituciones, de manera de que se alcance altos estándares de investigación e innovación.

Es necesario que las instituciones universitarias e instituciones gestoras de investigación presten especial atención a sus documentos publicados, igualmente, deben dar seguimiento a los indicadores que miden su productividad científica, y establecer estrategias que le permitan posicionarse dentro de los rankings SCImago. De este modo, el manejo de estos portales descritos en este artículo permitirá a los investigadores analizar la evolución y los adelantos que se tienen en cuanto desarrollo científico y tecnológico de las naciones.

\section{Referencias}

Arechavala, R. (2011). Las universidades y el desarrollo de la investigación científica y tecnológica en México: una agenda de investigación. Revista de la Educación Superior, 40(158), 41-57, eISSN: 0185-2760. Recuperado de:

http://www.scielo.org.mx/pdf/resu/v40n158/v40n158a3.pdf

De Moya-Anegón, F., Bustos-González, A., Chinchilla-Rodríguez, Z., CoreraÁlvarez, E., López-Illescas, C., \& Vargas-Quesada, B. (2014). Principales Indicadores Cienciométricos de la Actividad Científica Chilena 2012. Informe 2014: Una mirada a 10 años. ISBN: 123-9876543-21-0. Madrid, España: Comisión Nacional de Investigación 
Científica y Tecnológica CONICYT.

De Moya, F., Herrán-Páez, E., Bustos-González, A., Corera-Álvarez, E., Tibaná-Herrera, G., \& Rivadeneyra, F. (2019). Ranking Iberoamericano de instituciones de educación superior 2019 (SIR Iber). ISBN: 978-84-120239-1. Granada, España: El profesional de la información. Recuperado de: https://doi.org/10.3145/sir-iber-2019 Hernández, R., Fernández, C. \& Baptista, M. (2014). Metodología de la investigación. Sexta edición, ISBN: 978-1-4562-2396-0. México: McGraw-Hill / Interamericana Editores, S.A. de C.V.

Martínez, O. (2017). Cuando planteamos crear la Revista Scientific. Revista Scientific, 2(5), 6-7, e-ISSN: 2542-2987. Recuperado de:

https://doi.org/10.29394/scientific.issn.2542-2987.2017.2.5.0.6-7

Rodríguez, R., Socorro, A., \& Espinoza, C. (2019a,b,c,d). Análisis de Scimago Journal \& Country Rank, utilidad para el desarrollo bibliométrico en la Universidad Metropolitana del Ecuador. Revista Publicando, 6(21), 58-68, e-ISSN: 1390-9304. Recuperado de:

https://revistapublicando.org/revista/index.php/crv/article/view/1635

SIR (2020). SCImago Institutions Rankings. Spain: Scimago Lab.

Recovered from: http://www.scimagoir.com

SJR (2020a,b,c,d). SCImago Journal \& Country Rank. Spain: Scimago Lab.

Recovered from: http://www.scimagojr.com

Túñez-López, M., Valarezo-González, K., \& Marín-Gutiérrez, I. (2014).

Impacto de la investigación $y$ de los investigadores en comunicación en Latinoamérica: el índice $h$ de las revistas científicas. Palabra Clave, 17(3), 895-919, e-ISSN: 0122-8285. Recuperado de: https://doi.org/10.5294/pacla.2014.17.3.14 


\section{Eddymar María Flores Nessi}

e-mail: eddymarf.nessi@gmail.com

Nacida en la ciudad de Cabimas, Estado Zulia, Venezuela,

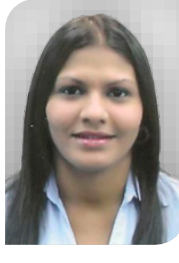
el 11 de marzo del año 1984. Ingeniera Química, Universidad del Zulia (LUZ, 2007); Magister en Gerencia de Proyectos de Investigación y Desarrollo, Universidad Dr. Rafael Belloso Chacín (URBE, 2016); Con experiencia docente a nivel universitario desde hace 10 años; Actualmente Docente a Tiempo Completo en la Universidad Politécnica Territorial del Zulia (UPTZ); Docente de las Unidades Curriculares: Proyectos, Ingeniería de las Reacciones Químicas, Ingeniera Ambiental; Tutora y jurado evaluador de trabajos y proyectos de Investigación; Experiencia en la asesoría y consultoría ambiental referida a la elaboración de estudios de impacto ambiental, diagnósticos ambientales y tramitación de autorizaciones ambientales. 


\section{Derwis Antonio Sulbarán Sandrea}

e-mail: dersulba@gmail.com

Nacido en la ciudad de Cabimas, Estado Zulia,

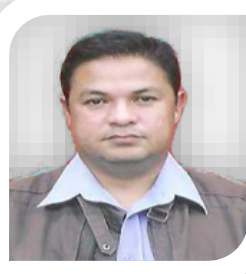
Venezuela, el 17 de mayo del año 1973. Licenciado en Trabajo Social; Magister en Docencia para la Educación Superior; Doctor en Educación; con experiencia docente desde hace más de 12 años; Actualmente Docente en la categoría de Asociado a Dedicación Exclusiva; Coordinador del Departamento de Postgrado; Coordinador de la Comisión de Formación para los Concursos Docentes en la Universidad Politécnica Territorial del Zulia (UPTZ); Tutor y jurado evaluador de trabajos de Investigación de Postgrado en la Universidad Nacional Experimental "Rafael María Baralt" (UNERMB). 


\section{Gisemar Ninoska Rojas de Ricardo \\ e-mail: gisemarninoska@gmail.com}

Nacida en la ciudad de Cabimas, Estado Zulia,

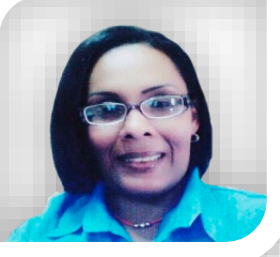
Venezuela, el 13 de febrero del año 1974. Licenciada en Educación Integral Mención Castellano, Literatura y Ciencias Biológicas de la Universidad Nacional Experimental "Rafael María Baralt" (UNERMB, 2000); Magíster Scientiarum en Administración de la Educación Básica de la mencionada casa de estudios (UNERMB, 2005); Docente con Función Directiva en la Escuela Básica Nacional "Don Rómulo Gallegos" desde febrero del año 2017.

El contenido de este manuscrito se difunde bajo una Licencia de Creative Commons ReconocimientoNoComercial-Compartirlgual 4.0 Internacional 